\title{
Phase Congruency Induced Local Features for
}

Finger-Knuckle-Print Recognition

\author{
Lin Zhang ${ }^{\mathrm{a}}$, Lei Zhang ${ }^{\mathrm{a}, *}$, David Zhang ${ }^{\mathrm{a}}$ and Zhenhua Guo ${ }^{\mathrm{b}}$ \\ ${ }^{a}$ Biometrics Research Center, Department of Computing, The Hong Kong Polytechnic University \\ ${ }^{\mathrm{b}}$ Graduate School at Shenzhen, Tsinghua University, China
}

\begin{abstract}
Researchers have recently found that the finger-knuckle-print (FKP), which refers to the inherent skin patterns of the outer surface around the phalangeal joint of one's finger, has high discriminability, making it an emerging promising biometric identifier. Effective feature extraction and matching plays a key role in such an FKP based personal authentication system. This paper studies image local features induced by the phase congruency model, which is supported by strong psychophysical and neurophysiological evidences, for FKP recognition. In the computation of phase congruency, the local orientation and the local phase, can also be defined and extracted from a local image patch. These three local features are independent of each other and reflect different aspects of the image local information. We compute efficiently the three local features under the computation framework of phase congruency using a set of quadrature pair filters. We then propose to integrate these three local features by score-level fusion to improve the FKP recognition accuracy. Such kinds of local features can also be naturally combined with Fourier transform coefficients, which are global features. Experiments are performed on the PolyU FKP database to validate the proposed FKP recognition scheme.
\end{abstract}

Keywords: Biometrics, finger-knuckle-print recognition, phase congruency

Corresponding author. Email: cslzhang@comp.polyu.edu.hk. This research is supported by the Hong Kong Polytechnic University ICRG (G-YH54) and the Ho Tung Fund (5-ZH52). 


\section{Introduction}

The need for reliable automated user authentication techniques has been significantly increased in the wake of heightened concerns about security [1]. Biometrics based methods, which use unique physical or behavioral characteristics of human beings, are drawing increasing attention in both academic research and industrial applications because of their high accuracy and robustness in the modern e-world. In the past decades, researchers have exhaustively investigated a number of different biometric identifiers, including fingerprint, face, iris, palmprint, hand geometry, voice, and gait, etc [2].

Among various kinds of biometric identifiers, hand-based biometrics attracts much interest because of their high user acceptance and convenience. Some commonly used hand-based biometrics, e.g., fingerprint $[3,4]$, palmprint $[5-11]$, hand geometry [12], and hand vein $[13,14]$, have been well investigated in the literature. Recently, scholars have reported that finger-knuckle-print (FKP), the image pattern of skin folds and creases in the outer finger knuckle surface, is highly unique and can serve as a distinctive biometric identifier [15-21]. Compared with fingerprint, FKP is hard to be abraded since people hold stuffs with the inner side of the hand. In addition, unlike fingerprint, there is no stigma of criminal investigation associated with the finger knuckle surface, so FKP can have a higher user acceptance [21]. Moreover, people rarely leave FKP remains on the stuff surface, making the loss of private data less possible. Thus, FKP has a great potential to turn into a widely accepted biometric identifier.

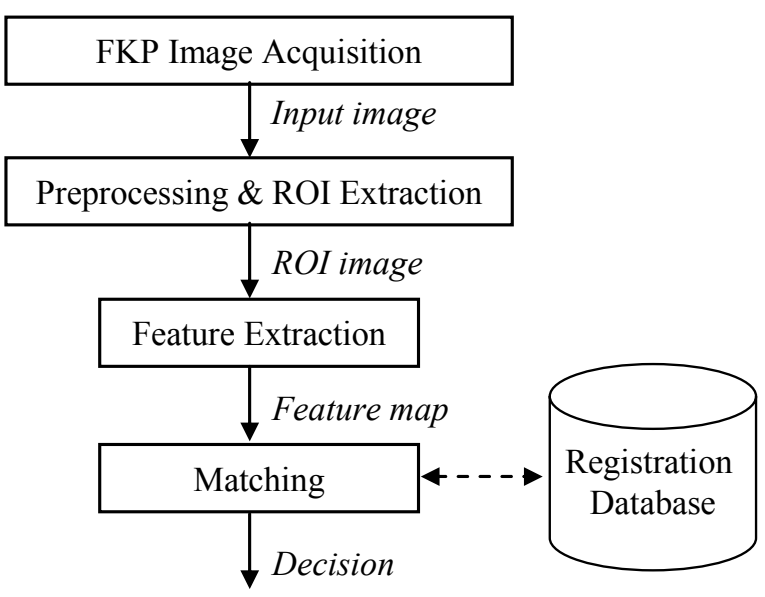

Fig. 1. Architecture of our FKP recognition system. 


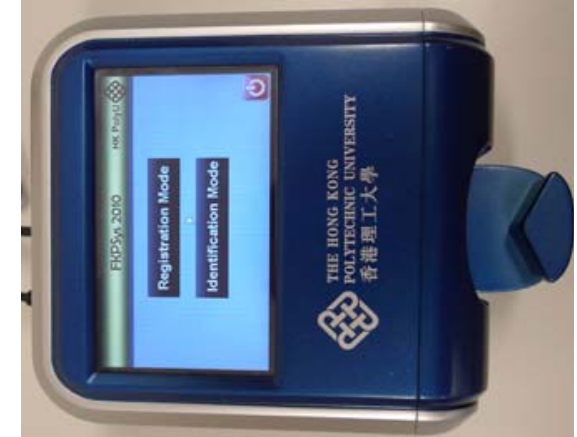

(a)

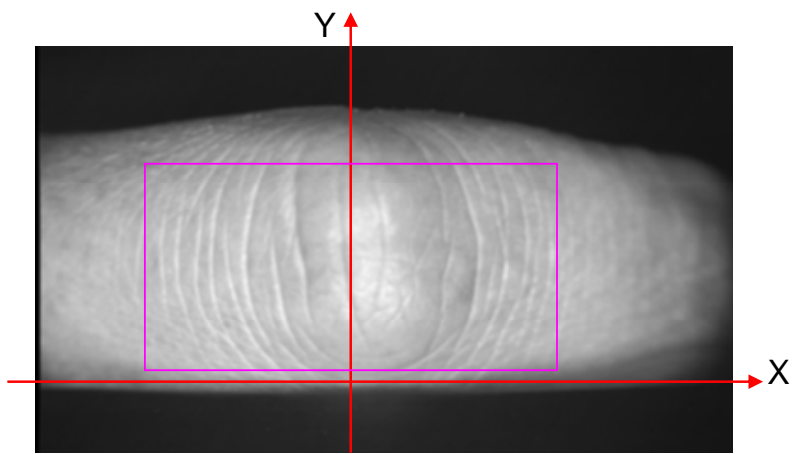

(c)

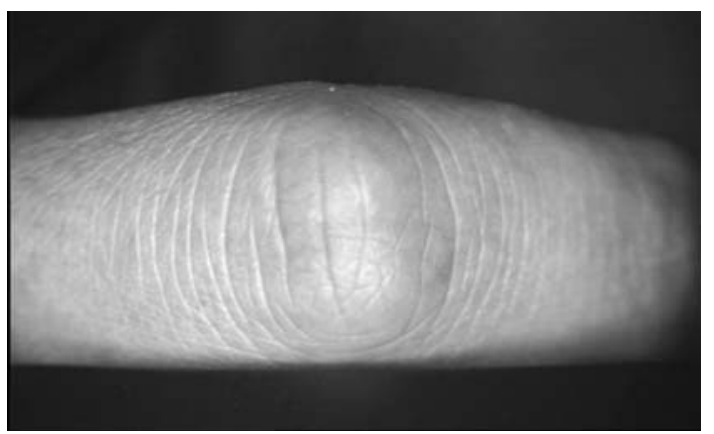

(b)

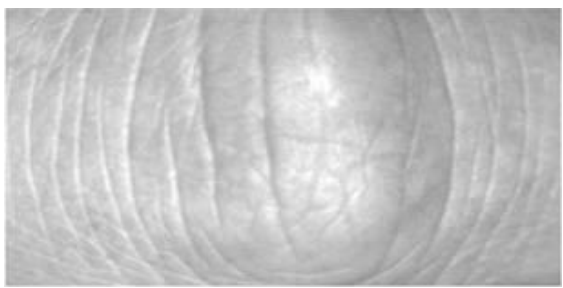

(d)

Fig. 2: (a) Outlook of our embedded FKP recognition system; (b) a typical FKP image; (c) the determination of ROI; (d) a cropped ROI image from the original FKP image in (c).

A novel online FKP-based personal authentication system has been established in our previous works [15-18]. As shown in Fig. 1, it comprises four major components: FKP image acquisition, ROI (region of interest) extraction, feature extraction and feature matching. In our design, the finger knuckle will be slightly bent when being imaged, and hence the inherent skin patterns can be clearly captured. Fig. 2a shows the outlook of our embedded FKP recognition system and Fig. 2b shows a typical FKP image. Fig. 2c and Fig. 2d illustrate the ROI extraction process presented in [15]. The feature extraction and matching are based on the extracted ROIs.

As in many pattern classification tasks, feature extraction and matching plays a key role in FKP-based personal authentication system. In [17], Zhang et al. used the Gabor filter based competitive coding scheme, which was originally designed for palmprint recognition [6], to extract the local orientation information as FKP features. In [15], Zhang et al. combined the orientation information and the magnitude information extracted by Gabor filters. In [18], the Fourier transform coefficients of the image were taken as the feature and the band-limited phase-only correlation technique was employed to calculate the similarity between two FKP images. In the local-global information combination (LGIC) feature extraction scheme [16], the local orientation extracted by the Gabor filters is taken as the local feature while the 
Fourier coefficients are taken as a global feature. In [19], Morales et al. used a real Gabor filter to enhance the FKP image and then used the scale invariant feature transform (SIFT) to extract features.

In our previous method $[15,17]$, real Gabor filters are used to extract the local orientation information; such an idea was inspired by the method [6] for palmprint recognition. Local orientation feature of biometric images can also be defined and extracted using other different mathematical models. For example, in [7], Jia et al. proposed a coding method to extract the local orientation of palmprints, namely robust line orientation code (RLOC), which is based on a modified finite Radon transform. In addition to the local orientation, the local phase is also widely used in the biometrics community and it is usually extracted by using band-pass complex filters, e.g., Gabor filters [22, 23] and log-Gabor filters [24]. By making use of the local phase feature extracted by Gabor filters, Daugman invented the famous IrisCode [25]; inspired by Daugman's work, Zhang et al. adopted a similar idea to match palmprint images [5]. Actually, according to $[26,27]$, the local phase reflects the type of local features. However, it is not clear whether such a feature is significant and stable. To address such an issue, we need to know whether the local phases over scales are consistent. The phase congruency (PC) model [28-31] serves as a solution to this issue. Studies of psychophysics and neurophysiology have revealed that visually discernable image features coincide with those points where Fourier waves at different frequencies have congruent phases. PC has been exploited as features by some biometrics researchers for face recognition [32], iris recognition [33] and palmprint recognition [8].

To the best of our knowledge, in the biometrics community there is no work to define and analyze systematically the three local features - local orientation, local phase, and PC - in a unified framework, and there is no attempt trying to integrate these three features to improve the performance of biometrics systems, either. In fact, these three local features are independent of each other and none of them can be covered by the others; they reflect different aspects of image local information. Thus, better performance could be expected by combining these three local features together in some way. Based on these considerations, in this paper, we first define these three local features under a unified framework, and then present an efficient method to compute them using the computation framework of PC. At last we integrate these three local features together for FKP recognition. Experimental results demonstrate that the integration of the three local features performs much better than using any of them separately. Moreover, we report the system's 
performance when integrating the three local features with one global feature, the Fourier transform coefficients, which leads to the best result on our benchmark FKP database.

The remainder of this paper is organized as follows. Section 2 defines and analyzes the three local features, while Section 3 presents the extraction and matching scheme for each local feature. Section 4 reports the experimental results and discussions. Finally, Section 5 concludes the paper.

\section{Analysis of Local Features}

As stated in Section 1, in literature the three local features, the local orientation, the local phase, and the phase congruency, are extracted by using different mathematical models and their relationships are not systematically investigated. In this section, we will examine these three local features in detail and under a unified framework.

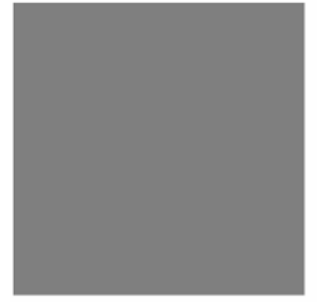

(a)

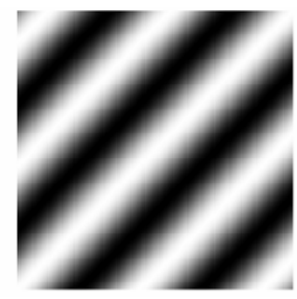

(b)

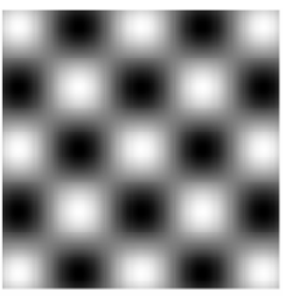

(c)

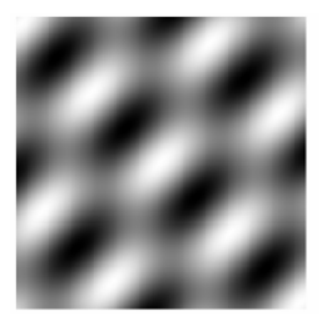

(d)

Fig. 3. Signals with different intrinsic dimensions. (a) i0D; (b) i1D; (c) i2D; (d) i2D.

To ease the following discussions, we first introduce the concept of intrinsic dimension here. The intrinsic dimension is the number of degrees of freedom necessary to describe a local image structure [34]. A 2D image patch can be classified into a local region, denoted by $R$, of different intrinsic dimension. For example, constant areas are of intrinsic dimension zero (i0D) while straight lines and edges are of intrinsic dimension one (i1D). Mathematically, such a classification can be expressed as

$$
f \in\left\{\begin{array}{l}
\mathrm{i} 0 D_{R}, f\left(\mathbf{x}_{i}\right)=f\left(\mathbf{x}_{j}\right), \forall \mathbf{x}_{i}, \mathbf{x}_{j} \in N \\
\mathrm{i} 1 D_{N}, f(x, y)=g(x \cos \theta+y \sin \theta), \forall(x, y) \in N, f \notin \mathrm{i} 0 D_{N} \\
\mathrm{i} 2 D_{N}, \text { else }
\end{array}\right.
$$

where $g$ is a 1D real-valued function. Examples of i0D, i1D, and i2D signals are shown in Fig .3. 
A point $\mathbf{x}$ in an image can be characterized by its "local features", which are derived from a local window centered on it. Before we define local features we need to have a model for the signal to be analyzed. In our case, we are dealing with 2D FKP images, which are actually a special kind of 2D images in that they are abundant of line-like features. And these line-like features play dominant roles in distinguishing different individuals. Thus, in this paper, we assume that FKP images are locally i1D (intrinsic one dimensional) signals.

Let's consider the one dimensional (1D) signal first. The analytic signal is an effective tool for 1D signal processing. Given a real 1D signal $f(x)$, the corresponding analytic signal is defined as

$$
f_{A}(x)=f(x)+i f_{H}(x)=f(x)+i(f(x) * h(x))
$$

where $h(x)=1 / \pi x$ refers to the Hilbert transform kernel in the spatial domain and $i^{2}=-1$. The local amplitude and the local phase of the 1D analytic signal are defined as

$$
a(x)=\sqrt{f^{2}(x)+f_{H}^{2}(x)}, \phi(x)=\arg f_{A}(x)
$$

The local amplitude indicates the energetic information of the signal, while the local phase can be used to distinguish between different local structures and it is independent of the local amplitude [26]. In practice, since the Hilbert transform operator is an improper integral and difficult to calculate, researchers usually use a pair of spatial filters forming a quadrature pair to construct the analytic signal $[26,35]$. To this end, complex Gabor $[22,23]$ or log-Gabor [24] filters are widely used. When the 1D signal is embedded into the 2D space, its orientation should be considered. Thus, the local amplitude, the local phase, and the local orientation are three independent measures to characterize a 2D image point.

The local phase reflects the type of local structures [ref]. However, we do not know to what degree it is a significant feature. To address such an issue, we make use of the phase congruency (PC) [28-30], a dimensionless quantity, to measure the consistency of the local phases over scales. Based on the physiological and psychophysical evidence, it is found that visually discernable features coincide with those points having maximal phase congruency. Such a conclusion has been further corroborated by some recent studies in neurobiology using functional magnetic resonance imaging (fMRI) [31]. Phase congruency has an intriguing property that it is almost invariant to changes in image brightness or contrast.

Thus, within the local window surrounding an image point $\mathbf{x}$, four features - the local amplitude, the 
local phase, the local orientation and the phase congruency, can be extracted and they reflect different aspects of information contained in the local window. However, we will not use the local amplitude for recognition because it is not contrast invariant. Hence, the local phase, the local orientation and the phase congruency will be used as three local features in this article.

For real 2D images, these three local features can be defined and extracted using a set of 2D quadrature filter pairs, such as 2D complex Gabor or log-Gabor filters. Suppose that complex Gabor filters are adopted, which are defined as

$$
G(x, y)=\exp \left(-\frac{1}{2}\left(\frac{x^{\prime 2}}{\sigma_{x}^{2}}+\frac{y^{\prime 2}}{\sigma_{y}^{2}}\right)\right) \cdot \exp \left(i \frac{2 \pi}{\lambda} x^{\prime}\right)
$$

where $x^{\prime}=x \cos \theta+y \sin \theta, y^{\prime}=-x \sin \theta+y \cos \theta$. In Eq. (4), $\lambda$ represents the wavelength of the sinusoid factor, $\theta$ represents the orientation of the normal to the parallel stripes of the Gabor function, $\sigma_{x}$ and $\sigma_{y}$ are the standard deviations of the 2D Gaussian envelop. It can be seen from the definition that a Gabor filter is actually a Gaussian envelop modulated by a sinusoidal plane wave. The Gaussian envelop ensures that the convolution is dominated by the image patch near the center of the filter. Therefore, the Gabor filter is a local operator and can extract information at a specific scale and a specific orientation within a local region.

To define and extract the local orientation, we make use of the competitive coding scheme which has been successfully used for palmprint [6] and FKP $[15,17]$ recognition. Denote by $G_{R}\left(G_{I}\right)$ the real (imaginary) part of the Gabor filter $G$. With a series of $G_{R}$ sharing the same parameters, except the parameter of orientation, the local orientation of the image $I$ at the position $(x, y)$ can be extracted. Mathematically, the local orientation is defined as

$$
\operatorname{ori}(x, y)=\underset{j}{\arg \min }\left\{I(x, y) * G_{R}\left(x, y, \theta_{j}\right)\right\}
$$

where $\theta_{j}=j \pi / J, j=\{0, \ldots, J-1\} . J$ represents the number of orientations. It needs to be noted that theoretically speaking, the local orientation of ideal i1D 2D image signals can be accurately extracted by the Riesz transform-based monogenic signal model, which is a $2 \mathrm{D}$ extension of the classical 1D analytic signal [36, 37]; however, for real 2D images, multi-dimensional even-symmetric filters usually perform better for this task $[6,15]$.

The extraction of the PC using quadrature pair filters will be presented in Section 3.1 in detail. Actually, 
the $\mathrm{PC}$ is a $1 \mathrm{D}$ concept. For $2 \mathrm{D}$ images, we can compute $P C_{\theta_{j}}$ along different orientations $\left\{\theta_{j}: \mid j=0 \sim J-1\right\}$.

Then the maximum of $\left\{P C_{\theta_{j}}: \mid j=0 \sim J-1\right\}$ can be taken as the PC value at the examined position:

$$
P C_{2}(x, y)=\max _{j}\left\{P C_{\theta_{j}}(x, y): \mid j=0 \sim J-1\right\}
$$

We denote by $\theta_{m}$ the orientation along which the 1D PC takes the maximum. Then, we can apply Gabor filtering along $\theta_{m}$ and define the local phase as:

$$
\text { phase }(x, y)=\arg \left(I(x, y) * G_{R}\left(x, y, \theta_{m}\right)+i I(x, y) * G_{I}\left(x, y, \theta_{m}\right)\right)
$$

\section{Extraction and Matching of Local Features}

In Section 2, we have defined and analyzed three local features. In practice, for the reason of computational efficiency, we do not compute the three local features separately. Instead, we present a scheme based on the computational framework of PC in [30] to extract those features more efficiently. So, in the following sub-sections, the PC its computation will be described first.

\subsection{Phase congruency (PC)}

Rather than assume a feature is a point of sharp changes in intensity, the PC model postulates that features are perceived at points where the Fourier components are maximal in phase [28-30]. Phase congruency can be considered as a dimensionless measure for the significance of a structure independently of the signal amplitude. The technique to calculate PC used in this paper is based on Kovesi's work [30].

We start from the 1D signal $f(x)$. Denote by $M_{n}^{e}$ and $M_{n}^{o}$ the even-symmetric and odd-symmetric filters at scale $n$ and they form a quadrature pair. Reponses of each quadrature pair to the signal will form a response vector at position $x$ and on scale $n$ :

$$
\left[e_{n}(x), o_{n}(x)\right]=\left[f(x) * M_{n}^{e}, f(x) * M_{n}^{o}\right]
$$

The local amplitude on scale $n$ is given by

$$
A_{n}(x)=\sqrt{e_{n}^{2}(x)+o_{n}^{2}(x)}
$$

and the local phase is given by 


$$
\phi_{n}(x)=\operatorname{atan} 2\left(o_{n}(x), e_{n}(x)\right)
$$

These response vectors form the basis of our localized representation of the signal and the PC can be derived from them.

Let $F(x)=\sum_{n} e_{n}(x)$ and $H(x)=\sum_{n} o_{n}(x)$. Then, the 1-D PC can be computed as

$$
P C(x)=\frac{E(x)}{\varepsilon+\sum_{n} A_{n}(x)}
$$

where $E(x)=\sqrt{F^{2}(x)+H^{2}(x)}$ and $\varepsilon$ is a small positive constant. We can also define the local phase as

$$
\operatorname{Phase}(x)=\operatorname{atan} 2(H(x), F(x))
$$

Actually, it is the average local phase over $n$ scales.

For 2D images, we have to apply the 1D analysis over several orientations and combine the results in some way. In such case, 2D filters with the orientation selection property can be used, such as the Gabor filters $[22,23]$ or $\log$-Gabor filters [24]. Let $\theta_{j}=j \pi / J, j=\{0,1, \ldots, J-1\}$, denote the orientation angle of the filter, where $J$ is the number of orientations. By modulating $n$ and $\theta_{j}$ and convolving with the 2D image, we can get a set of responses at each image point $\mathbf{x}$ as

$$
\left[e_{n, \theta_{j}}(\mathbf{x}), o_{n, \theta_{j}}(\mathbf{x})\right]
$$

The local amplitude of point $\mathbf{x}$ on scale $n$ and along orientation $\theta_{j}$ is given by

$$
A_{n, \theta_{j}}(\mathbf{x})=\sqrt{e_{n, \theta_{j}}(\mathbf{x})^{2}+o_{n, \theta_{j}}(\mathbf{x})^{2}}
$$

The local energy along orientation $\theta_{j}$ is given by

$$
E_{\theta_{j}}(\mathbf{x})=\sqrt{F_{\theta_{j}}(\mathbf{x})^{2}+H_{\theta_{j}}(\mathbf{x})^{2}}
$$

where $F_{\theta_{j}}(\mathbf{x})=\sum_{n} e_{n, \theta_{j}}(\mathbf{x})$ and $H_{\theta_{j}}(\mathbf{x})=\sum_{n} o_{n, \theta_{j}}(\mathbf{x})$. Then, the phase congruency along orientation $\theta_{j}$ is computed by

$$
P C_{\theta_{j}}(\mathbf{x})=\frac{E_{\theta_{j}}(\mathbf{x})}{\varepsilon+\sum_{n} A_{n, \theta_{j}}(\mathbf{x})}
$$

The average local phase along orientation $\theta_{j}$ is defined as 


$$
\operatorname{Phase}_{\theta_{j}}(\mathbf{x})=\operatorname{atan} 2\left(H_{\theta_{j}}(\mathbf{x}), F_{\theta_{j}}(\mathbf{x})\right)
$$

We define the 2D PC at $\mathbf{x}$ as

$$
P C_{2}(\mathbf{x})=\max _{j} P C_{\theta_{j}}(\mathbf{x})
$$

It should be noted that $P C_{2}(\mathbf{x})$ is a real number within $0 \sim 1$.

\subsection{Local feature extraction and coding}

In this section, we present the extraction and coding algorithm for each local feature. The local orientation and the local phase can be efficiently extracted by using the intermediate results of the PC computation.

Having obtained two raw PC maps of two images, we do not match them directly. Instead, we quantize them to several levels and then code them into integers. In practice, such a scheme can have three advantages: a) it can save a lot the storage space; b) for recognition, it works more robustly than using raw PC maps; and c) it allows a fast matching of two maps. Therefore, we quantize the PC into $L$ levels and define the PC code as

$$
\operatorname{pcCode}(\mathbf{x})=\left\lfloor\frac{P C_{2}(\mathbf{x})}{1 / L}\right\rfloor
$$

where $\lfloor x\rfloor$ is the operator to return the largest integer not bigger than $x$. It is easy to see that each $p c$ Code is an integer within $0 \sim L-1$.

Although there are other kinds of methods to evaluate the local phase feature and the local orientation feature, we want to make a full use of the intermediate results in the process of computing PC in order to reduce the computational cost. It is easy to see that when calculating the $\mathrm{PC}$, we can get responses from a set of even-symmetric and odd-symmetric quadrature filters at different scales and different orientations. We can compute the local orientation and the local phase directly from them. For the local orientation evaluation, we borrow the idea from the competitive coding scheme $[6,15,17]$. With the responses from the even-symmetric filters at a certain scale $\varsigma$, i.g. $\left\{e_{\zeta, \theta_{j}}(\mathbf{x}): j=0 \sim J-1\right\}$, the orientation code at $\mathbf{x}$ can be defined as

$$
\operatorname{oriCode}(\mathbf{x})=\underset{j}{\arg \min }\left\{e_{\zeta, \theta_{j}}(\mathbf{x})\right\}, j=0, \ldots, J-1
$$


Obviously, each orientation code oriCode $(\mathbf{x})$ is an integer within $0 \sim J-1$.

Refer to Eq. (18), by our definition the 2D PC is actually the maximum of the 1D PCs along different orientations. We denote by $\theta_{m}$ the orientation along which the 1D PC takes the maximum value. Then, we can take the average local phase along $\theta_{m}$ as the local phase at $\mathbf{x}$. That is

$$
L P(\mathbf{x})=\text { Phase }_{\theta_{m}}(\mathbf{x})
$$

The range of $L P$ is $[0,2 \pi]$. Once again, we do not need the exact local phase angle. Instead, we quantize $L P$ into several discrete levels to get the "phase code" as

$$
\operatorname{phaCode}(\mathbf{x})=\lfloor L P(\mathbf{x}) /(2 \pi / M)\rfloor
$$

where $M$ is the number of quantization levels. Thus, each phase code is an integer within $0 \sim M-1$.

Finally, for a given image, we can get its three code maps: pcCode, oriCode, and phaCode. Examples of them are shown in Fig. 4.

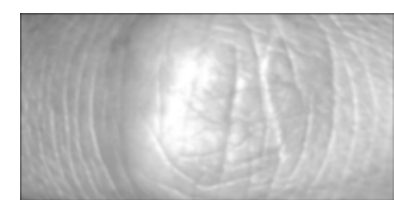

$\left(a_{1}\right)$

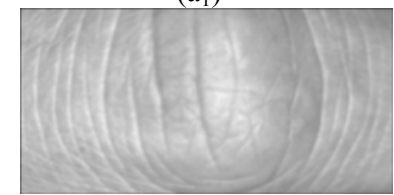

$\left(a_{2}\right)$

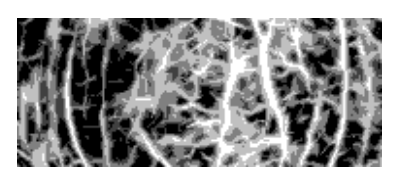

$\left(b_{1}\right)$

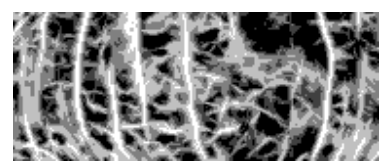

$\left(b_{2}\right)$

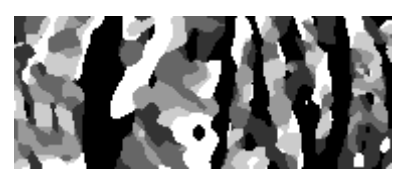

$\left(c_{1}\right)$

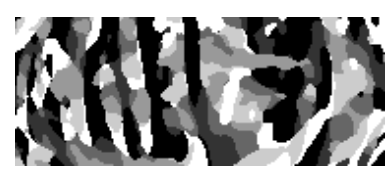

$\left(c_{2}\right)$

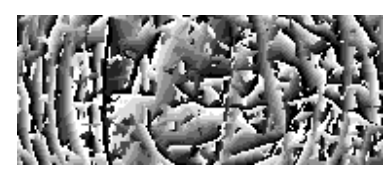

$\left(d_{1}\right)$

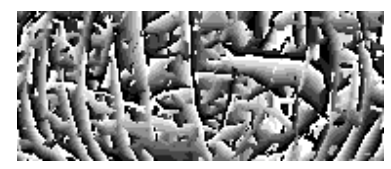

$\left(d_{2}\right)$

Fig. 4. Examples of local feature maps. $\left(a_{1}\right)$ and $\left(a_{2}\right)$ are the original FKP ROI images; $\left(b_{1}\right)$ and $\left(b_{2}\right)$ are the corresponding pcCode maps; $\left(\mathrm{c}_{1}\right)$ and $\left(\mathrm{c}_{2}\right)$ are the corresponding oriCode maps; $\left(\mathrm{d}_{1}\right)$ and $\left(\mathrm{d}_{2}\right)$ are the corresponding phaCode maps.

\subsection{Matching of local feature maps}

Having obtained the three code maps pcCode, oriCode, and phaCode for each image, the next issue is how to match them for recognition. Since the PC is a dimensionless measure, we can use the absolute difference to measure the distance between two pcCode maps. Specifically, given two PC code maps, $p c C o d e 1$ and $p c \operatorname{Code2}$, we define their normalized matching distance as

$$
p c D=\frac{\sum \sum a b s(p c \operatorname{Code} 1(\mathbf{x})-p c \operatorname{Code} 2(\mathbf{x}))}{(L-1) \cdot S}
$$

where $S$ is the area of the image. 
For comparing two orientation code maps, oriCode 1 and oriCode 2 , we resort to the normalized angular distance proposed in [6], which is defined as

$$
\begin{aligned}
& \operatorname{oriD}=\frac{\sum \sum \operatorname{ang}(\text { oriCode } 1(\mathbf{x}), \text { oriCode } 2(\mathbf{x}))}{S \cdot J / 2} \\
& \operatorname{ang}(p, q)=\left\{\begin{array}{l}
\min (p-q, q-p+J), p \geq q \\
\min (q-p, p-q+J), p<q
\end{array}\right.
\end{aligned}
$$

When matching two phase code maps, we use a similar method as matching two orientation code maps. The matching distance between two phase code maps, phaCode 1 and phaCode2, is given by

$$
\text { phaD }=\frac{\sum \sum \operatorname{ang}(\text { phaCode1 }(\mathbf{x}), \text { phaCode } 2(\mathbf{x}))}{S \cdot M / 2}
$$

In real implementation, it is easy to design "bitwise" representations for pcCode, oriCode and phaCode, and accordingly, $p c D$, oriD and phaD can be effectively computed.

\subsection{Integration of local features}

The three local features reflect different aspects of information contained in an image patch. Thus, we can expect higher recognition accuracy when assembling information from the three features together. This can be achieved by a score-level fusion and we refer to this feature integration scheme as local feature integration (LFI) in this paper. Suppose that three matching distances $p c D$, oriD, and $p h a D$ have been calculated by matching the three kinds of local features respectively. These three distances can be fused together to get the final matching distance. There are a couple of rules for the fusion of matching distances, such as the Simple-Sum (SS) rule, the MIn-Score (MIS) rule, the MAx-Score (MAS) rule, and the Matcher-Weighting (MW) rule [38]. In our case, $p c D$, oriD, and $p h a D$ can be considered to be obtained from three different matchers and we adopt the MW rule. With the MW fusion rule, weights are assigned according to the Equal Error Rate (EER) obtained on a training dataset by different matchers. Denote by $e_{k}$ the EER of the matcher $k, k=1, \ldots, 3$. Then, the weight $w_{k}$ associated with matcher $k$ can be calculated as

$$
w_{k}=\left(1 / \sum_{j=1}^{3} \frac{1}{e_{j}}\right) / e_{k}
$$

where $0 \leq w_{k} \leq 1$ and $\sum_{k=1}^{3} w_{k}=1$. It is obvious that the weights are inversely proportional to the corresponding 
EERs. Then, the final matching distance between two FKP images using LFI is calculated as

$$
d=w_{1} p c D+w_{2} \text { oriD }+w_{3} p h a D
$$

\section{Experimental Results and Discussions}

\subsection{FKP database and the test protocol}

In our previous work [15-18], an FKP database was established using the developed FKP image acquisition device. This database is intended to be a benchmark to evaluate the performance of various FKP recognition methods, and it is publicly online available at [39]. In this database, FKP images were collected from 165 volunteers, including 125 males and 40 females. Among them, 143 subjects were 20 30 years old and the others were $30 \sim 50$ years old. We collected samples in two separate sessions. In each session, the subject was asked to provide 6 images for each of the left index finger, the left middle finger, the right index finger and the right middle finger. Therefore, 48 images from 4 fingers were collected from each subject. In total, the database contains 7,920 images from 660 different fingers. The average time interval between the first and the second sessions was about 25 days. The maximum and minimum time intervals were 96 days and 14 days respectively. In all of the following experiments, we took images collected at the first session as the gallery set and images collected at the second session as the probe set. To obtain statistical results, each image in the probe set was matched with all the images in the gallery set. If the two images were from the same finger, the matching between them was counted as a genuine matching; otherwise it was counted as an imposter matching.

The equal error rate (EER), which is the point where the false accept rate (FAR) is equal to the false reject rate (FRR), is used to evaluate the verification accuracy. The decidability index $d^{\prime}[40]$ is used to measure how well the genuine and the imposter distributions are separated. $d^{\prime}$ is defined as

$$
d^{\prime}=\frac{\left|\mu_{1}-\mu_{2}\right|}{\sqrt{\left(\sigma_{1}^{2}+\sigma_{2}^{2}\right) / 2}}
$$

where $\mu_{1}\left(\mu_{2}\right)$ is the mean of the genuine (imposter) matching distances and $\sigma_{1}\left(\sigma_{2}\right)$ is the standard deviation of the genuine (imposter) matching distances. Besides, by adjusting the matching threshold, a detection 
error tradeoff (DET) curve [41], which is a plot of false reject rate (FRR) against false accept rate (FAR) for all possible thresholds, can be created. The DET curve can reflect the overall verification accuracy of a biometric system. Thus, the DET curve obtained by using each evaluated method will be provided.

\subsection{Determination of parameters}

In real implementation, with respect to the quadrature pair filters, we utilized the log-Gabor filters whose transfer function in the frequency domain is

$$
G_{2}\left(\omega, \theta_{j}\right)=\exp \left(-\frac{\left(\log \left(\omega / \omega_{0}\right)\right)^{2}}{2 \sigma_{r}^{2}}\right) \cdot \exp \left(-\frac{\left(\theta-\theta_{j}\right)^{2}}{2 \sigma_{\theta}^{2}}\right)
$$

where $\omega_{0}$ is the filter's center frequency, $\sigma_{r}$ controls the filter's radial bandwidth and $\sigma_{\theta}$ determines the filter's angular bandwidth. In the spatial domain, a log-Gabor filter has a similar shape with a Gabor filter [30]. However, compared with Gabor filters, log-Gabor filters have some special advantages [24, 30]. At first, one cannot construct Gabor filters of arbitrary bandwidth and still maintain a reasonably small DC component in the even-symmetric filter, while log-Gabor filters, by definition, has no DC component. Secondly, the transfer function of the log-Gabor filter has an extended tail at the high frequency end, which makes it more capable to encode natural images than ordinary Gabor filters. Thus, we chose to use log-Gabor filters to compute local features discussed in Sections 2 and 3 in this paper. Parameters were empirically tuned based on a sub-dataset containing images from the first 300 FKP classes and the tuning criterion was that parameter values that could lead to a lower EER would be chosen. As a result, parameters were set as the following: $n=3, J=6, \sigma_{\theta}=0.44, L=5, \varsigma=3, M=8, \omega_{0}^{1}=0.60, \omega_{0}^{2}=0.167, \omega_{0}^{3}=0.083$, where $\omega_{0}^{1}, \omega_{0}^{2}$ and $\omega_{0}^{3}$ represent the three center frequencies of the log-Gabor filters at three scales.

\subsection{Performance of local features}

In this experiment, we validate our claim that LFI could provide higher performance than using any of the three local features (the local orientation, the local phase, and the phase congruency) individually. In this experiment, all the classes of FKPs were involved. Therefore, there were $660(165 \times 4)$ classes and 3,960 $(660 \times 6)$ images in the gallery set and the probe set each. Each image in the probe set was matched against 
all the images in the gallery set. Thus, the numbers of genuine matchings and imposter matchings were 23,760 and $15,657,840$, respectively.

The verification accuracy by using each single feature, the local orientation, the local phase, or the PC, is given in Table 1. The performance of the LFI scheme is also reported in Table 1. The performance of state-of-the-art FKP recognition method, CompCode [15], is listed in Table 1 for comparison. The DET curves obtained by the evaluated methods are shown in Fig. 5.

From the experimental results shown in Table 1 and Fig. 5, we can have the following findings. At first, the local orientation can provide higher discriminability than the other two local features, the local phase and the PC, for the task of FKP verification. Secondly, "local orientation" and "CompCode" have nearly the same performance because both of them exploit the orientation information. In fact, the local orientation in this paper is extracted by using log-Gabor filters while CompCode extracts such information by using Gabor filters. Thus, we can conclude that Gabor filters and log-Gabor filters have very similar performance for orientation feature extraction. Thirdly, the LFI scheme which integrates all the three local features together performs obviously better than using any of them individually, which corroborates our claim.

It should be noted that LFI has higher computational cost and needs more storage space than CompCode [15]. At the feature extraction stage, the major operations involved are convolutions. So, the number of convolutions used can roughly reflect the overall computational complexity of the feature extraction. For CompCode, 6 convolutions are needed [15]. For LFI, in order to compute the phase congruency, at each specific scale 6 log-Gabor filtering are applied and altogether 3 scales are adopted; thus, $6 \times 3=18$ convolutions are needed. Therefore, LFI has about 3 times the computational complexity of CompCode. In CompCode, each feature point is represented by 3-bits. In LFI, for each feature point, 3-bits are used to represent each local feature, and thus 9-bits are used to represent a feature point. So, it is easy to see that LFI needs 3 times storage space compared with CompCode.

Table 1: Performance of different FKP verification schemes based on local feature(s)

\begin{tabular}{ccc}
\hline feature type & EER $(\%)$ & $d^{\prime}$ \\
\hline local orientation & 1.67 & 4.2847 \\
local phase & 3.01 & 2.9213 \\
local phase congruency & 2.59 & 3.3811 \\
CompCode [15] & 1.66 & 4.2989 \\
LFI & 1.27 & 4.3221 \\
\hline
\end{tabular}




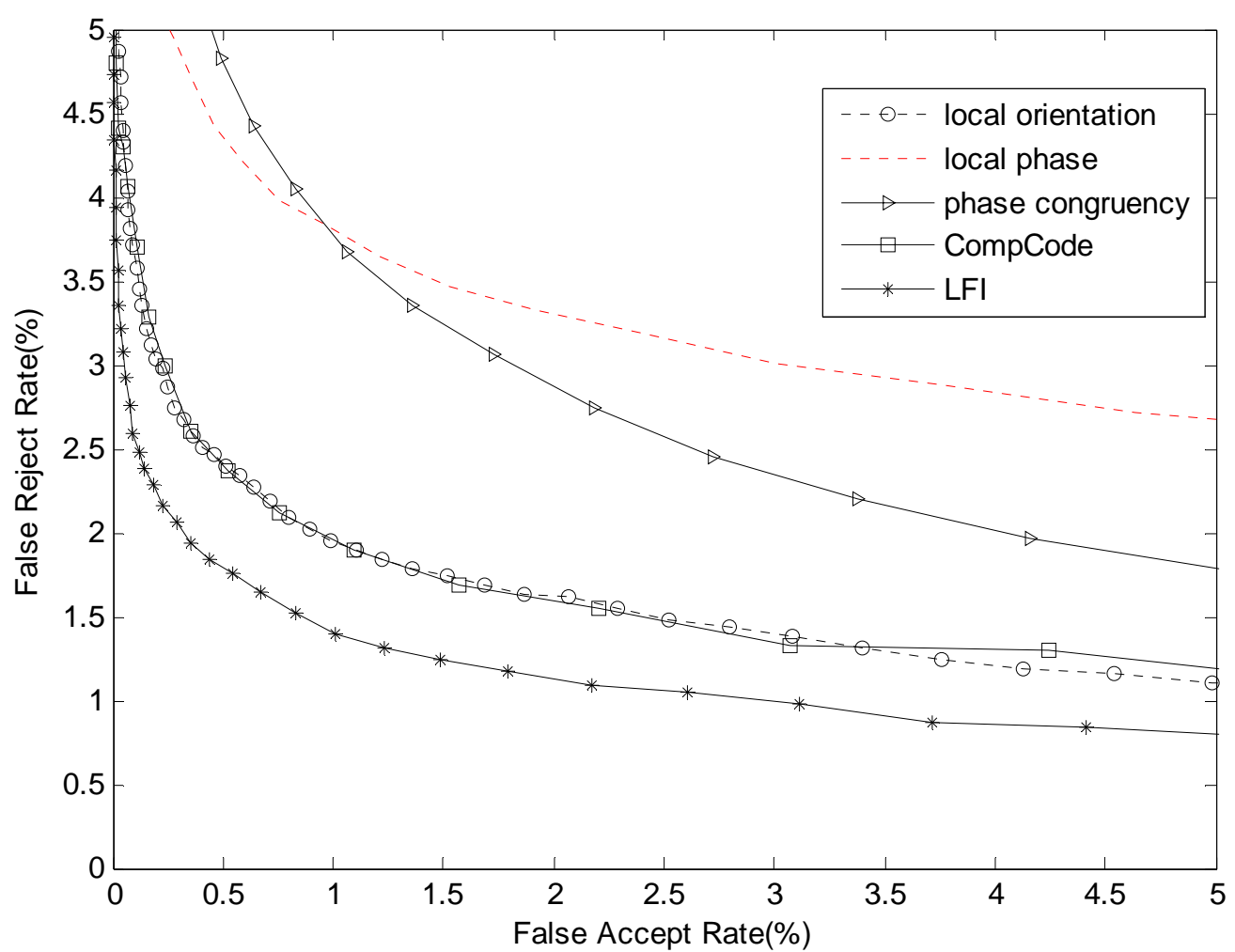

Fig. 5. DET curves obtained by using various FKP recognition methods based on local feature(s).

\subsection{Further discussions}

\subsubsection{Robustness to small rotations}

From the experimental results reported in Section 4.3, we can see that the local orientation has much higher discriminability than the local phase and PC for FKP recognition, and by incorporating the local phase and PC, the verification performance could be much better than using the local orientation feature alone. The local phase and PC could provide additional discriminative information that is independent of local orientation for the FKP verification task. In addition, the local phase and PC features are more robust to small rotations than the local orientation. Due to the imperfection of the imaging device and the ROI extraction algorithm, there will be small rotations among intra-class images captured at different times, which will have negative effect to the algorithms mainly depending on the orientation information. To validate such a conjecture, we tested the robustness to small rotations of each local feature in this section.

For this purpose, we selected images from the first 400 FKP classes as the dataset and the experiment protocols were the same as described in Section 4.1. Let $\alpha=\{0,1,2,3,4,5\}$. For each $\alpha$, by rotating each 
image in the dataset randomly by a degree within range $[-\alpha, \alpha]$, we can get a new virtual dataset. The verification performances of each local feature in terms of EER on these 6 virtual datasets are summarized in Table 2. For comparison, we also list the results obtained by CompCode and LFI under the same experimental settings in Table 2.

Table 2. Verification performance (measured by EER) of local features on the virtual rotated datasets

\begin{tabular}{ccccccc}
\hline & $\alpha=0$ & $\alpha=1$ & $\alpha=2$ & $\alpha=3$ & $\alpha=4$ & $\alpha=5$ \\
\hline local orientation & $2.03 \%$ & $2.04 \%$ & $2.16 \%$ & $2.40 \%$ & $2.78 \%$ & $3.61 \%$ \\
local phase & $3.63 \%$ & $3.65 \%$ & $3.87 \%$ & $4.21 \%$ & $4.64 \%$ & $5.50 \%$ \\
phase congruency & $2.83 \%$ & $2.86 \%$ & $2.91 \%$ & $3.23 \%$ & $3.36 \%$ & $3.88 \%$ \\
CompCode [15] & $2.01 \%$ & $2.03 \%$ & $2.15 \%$ & $2.38 \%$ & $2.77 \%$ & $3.60 \%$ \\
LFI & $1.52 \%$ & $1.54 \%$ & $1.64 \%$ & $1.77 \%$ & $1.96 \%$ & $2.34 \%$ \\
\hline
\end{tabular}

As shown in Table 2, when $\alpha$ increases, the EER obtained by using each local feature increases. However, for different local features, the "accelerations" of the EER increase are different. We use the following measure to characterize the "acceleration" of the EER increase

$$
\eta=\frac{1}{5} \sum_{i=1}^{5} \frac{e e r_{i}-e e r_{0}}{e e r_{0}}
$$

where $e e r_{i}$ is the EER obtained when $\alpha=i$. By using this metric $\eta$, the "acceleration" of the EER increase by using different local features while $\alpha$ increases can be compared. For the local orientation, $\eta=0.2798$; for the local phase $\eta=0.2050$; and for the PC, $\eta=0.1477$. From this experiment, we can clearly see that with the increase of the rotation degree, the performance decrease of local phase and PC is much less than local orientation, which indicates that the local phase and PC are more robust to small rotations than the local orientation. Moreover, for CompCode, $\eta=0.2866$ and for LFI, $\eta=0.2171$, which indicates that the proposed local feature integration scheme LFI is much more robust to small rotations than the CompCode scheme which depends on local orientation information only.

\subsubsection{Integrating local features with global feature}

In our previous work [16], we presented a local-global information combination (LGIC) scheme for FKP recognition, in which the local orientation extracted by Gabor filters was taken as the local feature while the image's Fourier transform coefficients were taken as the global feature. Similarity of Fourier transform 
coefficients from two images were compared using the phase-only correlation (POC) technique [42, 43]. LGIC could achieve the highest verification accuracy on our FKP database. In fact, the local features discussed in this paper can also be integrated with the global feature, i.e., the Fourier transform coefficients, using the same framework as LGIC. We call this new local-global information combination scheme as $\mathrm{LGIC}_{2}$. Compared with LGIC, $\mathrm{LGIC}_{2}$ involves two more local features, the local phase and the phase congruency. We compared the performance of $\mathrm{LGIC}$ and $\mathrm{LGIC}_{2}$ under the same experiment settings as described in Section 4.3. The results in terms of EER and $d^{\prime}$ are summarized in Table 3. DET curves obtained by LGIC and $\mathrm{LGIC}_{2}$ are shown in Fig. 6a. Distance distributions of genuine machings and imposter matchings obtained by $\mathrm{LGIC}_{2}$ are plotted in Fig. 6b. From the experimental results, we can see that $\mathrm{LGIC}_{2}$ performs better than LGIC. It once again corroborates our claim that the local phase and the phase congruency could afford more discriminative information for FKP recognition.

Table 3. FKP verification performance of $\mathrm{LGIC}$ and $\mathrm{LGIC}_{2}$

\begin{tabular}{ccc}
\hline Method & EER (\%) & $d^{\prime}$ \\
\hline LGIC [16] & 0.402 & 4.5356 \\
LGIC $_{2}$ & $\mathbf{0 . 3 5 8}$ & $\mathbf{4 . 7 0 0 1}$ \\
\hline
\end{tabular}

\section{Conclusions}

In this paper, we focused on developing new effective feature extraction and matching method for FKP recognition. To this end, we analyzed three commonly used local features, the local orientation, the local phase, and the phase congruency systematically and presented a method for computing them efficiently using the phase congruency computation framework. Coding and matching algorithm for each local feature was presented. Extensive experiments were conducted on the benchmark PolyU FKP database. The experimental results showed that the integration of all the local features together performs better than using any of them separately. The algorithm $\mathrm{LGIC}_{2}$, which integrates all the three local features and one global feature, Fourier transform coefficients, could achieve the best verification result on the benchmark FKP database, with the EER being $0.358 \%$. 


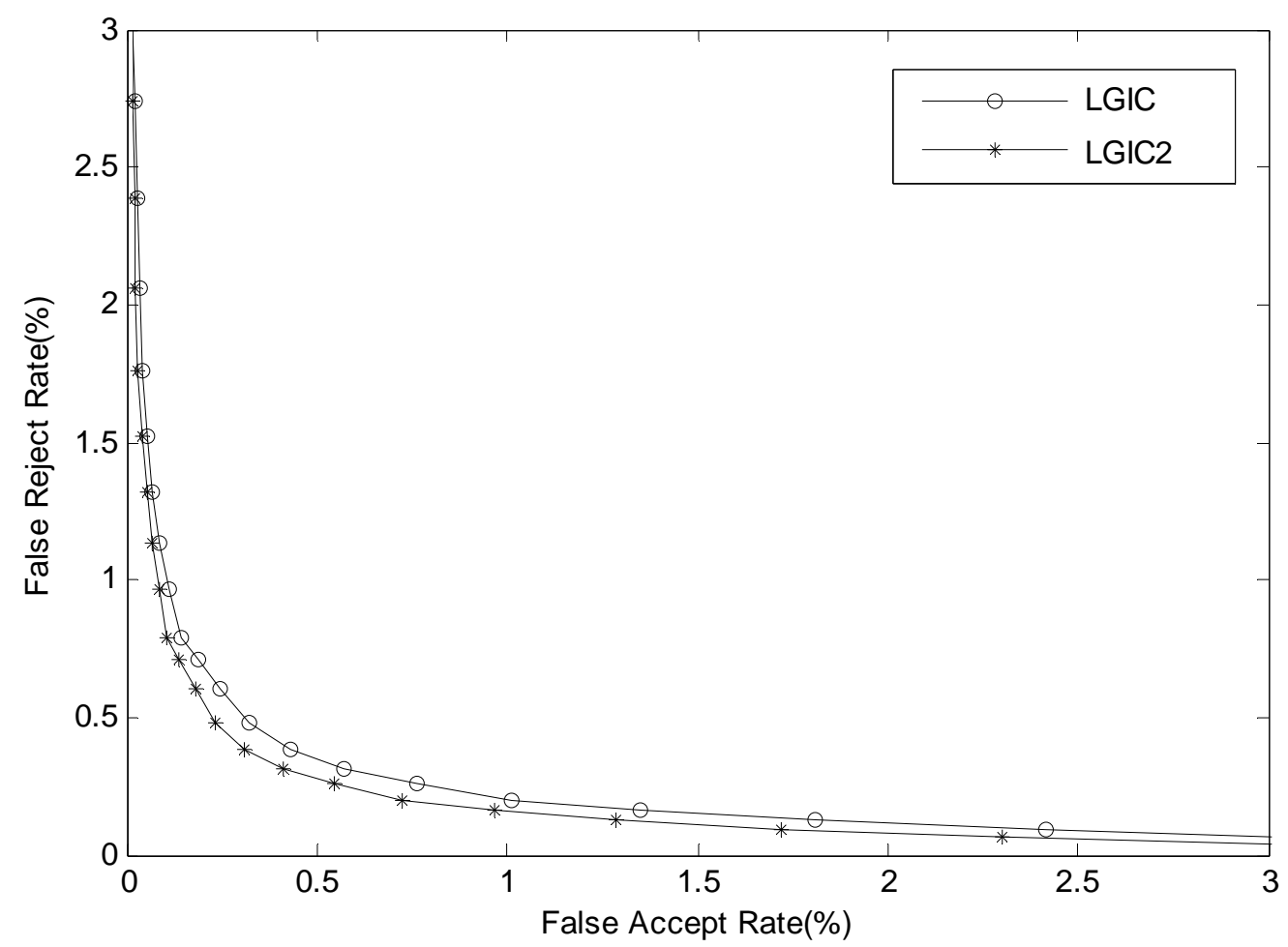

(a)

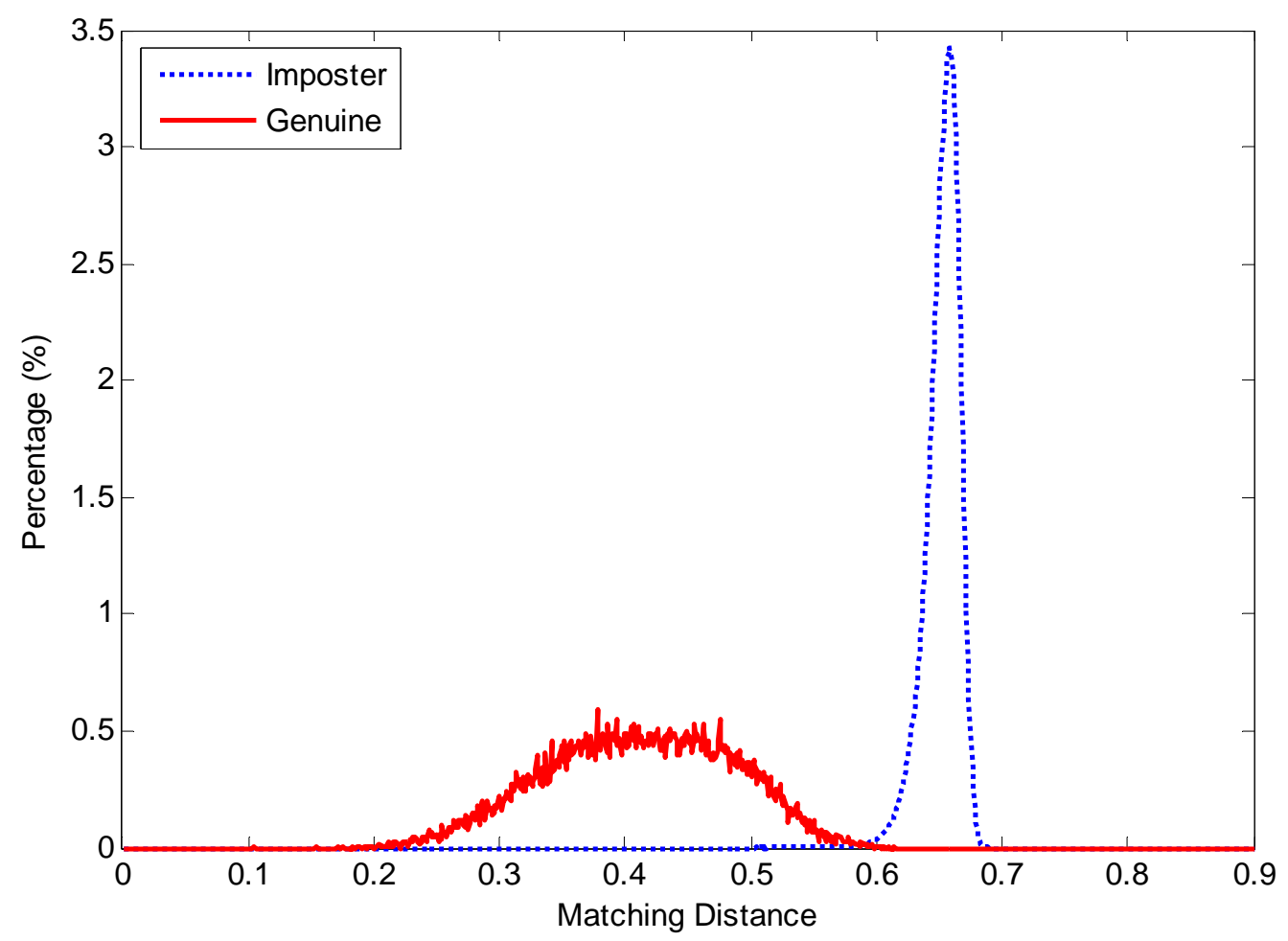

(b)

Fig. 6. (a) DET curves obtained by LGIC and $\mathrm{LGIC}_{2}$; (b) distance distributions of genuine matchings and imposter matchings obtained by $\mathrm{LGIC}_{2}$. 


\section{References}

[1] A.K. Jain, P.J. Flynn, A. Ross, Handbook of Biometrics, Springer, 2007.

[2] S.Z. Li (Ed.), Encyclopedia of Biometrics, Springer, 2009.

[3] L. Hong, Y. Wan, A. Jain, Fingerprint image enhancement: algorithm and performance evaluation, IEEE Transactions on Pattern Analysis and Machine Intelligence 20 (8) (1998) 777-789.

[4] D. Maltoni, D. Maio, A.K. Jain, S. Prabhakar, Handbook of Fingerprint Recognition, Springer, 2003.

[5] D. Zhang, W. Kong, J. You, M. Wong, Online palmprint identification, IEEE Transactions on Pattern Analysis and Machine Intelligence 25 (9) (2003) 1041-1050.

[6] A. Kong, D. Zhang, Competitive coding scheme for palmprint verification, in: Proceedings of the ICPR'04, 2004, pp. 520-523.

[7] W. Jia, D. Huang, D. Zhang, Palmprint verification based on robust line orientation code, Pattern Recognition 41 (5) (2008) 1504-1513.

[8] V. Struc, N. Pavesic, Phase congruency features for palm-print verification, IET Signal Processing 3 (4) (2009) $258-268$

[9] A.K. Jain, J. Feng, Latent palmprint matching, IEEE Transactions on Pattern Analysis and Machine Intelligence 31 (6) (2009) 1032-1047.

[10]A. Kong, D. Zhang, M. Kamel, A survey of palmprint recognition, Pattern Recognition 42 (7) (2009) 1408-1418.

[11]D. Zhang, W. Zuo, F. Yue, A comparative study of palmprint recognition algorithms, ACM Computing Surveys, to appear.

[12]N. Duta, A survey of biometric technology based on hand shape, Pattern Recognition 42 (11) (2009) 2797-2806.

[13] J. Wang, W. Yau, A. Suwandy, E. Sung, Personal recognition by fusing palmprint and palm vein images based on “Laplacianpalm” representation, Pattern Recognition 41 (5) (2008) 1531-1544.

[14]C. Wilson, Vein Pattern Recognition, CRC Press, 2010.

[15]L. Zhang, L. Zhang, D. Zhang, H. Zhu, Online finger-knuckle-print verification for personal authentication, Pattern Recognition 43 (7) (2010) 2560-2571.

[16]L. Zhang, L. Zhang, D. Zhang, H. Zhu, Ensemble of local and global information for finger-knuckle-print recognition, Pattern Recognition, to appear.

[17]L. Zhang, L. Zhang, D. Zhang, Finger-knuckle-print: a new biometric identifier, in: Proceedings of the ICIP'09, 2009, pp. 1981-1984. 
[18]L. Zhang, L. Zhang, D. Zhang, Finger-knuckle-print verification based on band-limited phase-only correlation, in: Proceedings of the CAIP'09, 2009, pp. 141-148.

[19] A. Morales, C.M. Travieso, M.A. Ferrer, J.B. Alonso, Improved finger-knuckle-print authentication based on orientation enhancement, Electronics Letters 47 (6) (2011) 380-381.

[20] D.L. Woodard, P.J. Flynn, Finger surface as a biometric identifier, Computer Vision and Image Understanding 100 (3) (2005) 357-384.

[21]A. Kumar, C. Ravikanth, Personal authentication using finger knuckle surface, IEEE Transactions on Information Forensics and Security 4 (1) (2009) 98-109.

[22]D. Gabor, Theory of communication, Journal of Institution of Electrical Engineers 93 (III) (1946) 429-457.

[23]J.G. Daugman, Uncertainty relation for resolution in space, spatial frequency, and orientation optimized by two-dimensional visual cortical filters, Journal of the Optical Society of America A 2 (7) (1985) 1160-1169.

[24]D.J. Field, Relations between the statistics of natural images and the response properties of cortical cells, Journal of the Optical Society American A 4 (12) (1987) 2379-2394.

[25] J.G. Daugman, High confidence visual recognition of persons by a test of statistical independence, IEEE Transactions on Pattern Analysis and Machine Intelligence 15 (11) (1993) 1148-1161.

[26] S. Venkatesh, R. Owens, On the classification of image features, Pattern Recognition Letters 11 (5) (1990) $339-349$.

[27] V. Sierra-Vazquez, I. Serrano-Pedraza, Application of Riesz transforms to the isotropic AM-PM decomposition of geometrical-optical illusion images, Journal of the Optical Society of America A 27 (4) (2010) 781-796.

[28] M.C. Morrone, J. Ross, D.C. Burr, R. Owens, Mach bands are phase dependent, Nature 324 (6049) (1986) 250-253.

[29] M.C. Morrone, D.C. Burr, Feature detection in human vision: a phase-dependent energy model, Proc. R. Soc. Lond. B 235 (1280) (1988) 221-245.

[30]P. Kovesi, Image features from phase congruency, Videre: Journal of Computer Vision Research 1 (3) (1999) $1-26$.

[31]L. Henriksson, A. Hyvärinen, S. Vanni, Representation of cross-frequency spatial phase relationships in human visual cortex, Journal of Neuroscience 29 (45) (2009) 14342-14351.

[32] S. Gundimada, V.K. Asari, A novel neighborhood defined feature selection on phase congruency images for recognition of faces with extreme variations, International Journal of Information Technology 3 (1) (2007) 25-31. 
[33]X. Yuan, P. Shi, Iris feature extraction using 2D phase congruency, in: Proceedings of the ICITA'05, 2005, pp. $437-441$.

[34] G. Krieger, C. Zetzsche, Nonlinear image operators for the evaluation of local intrinsic dimensionality, IEEE Transactions on Image Processing 5 (6) (1996) 1026-1042.

[35] M.C. Morrone, R. Owens, Feature detection from local energy, Pattern Recognition Letters 6 (5) (1987) $303-313$.

[36] M. Felsberg, G. Sommer, The monogenic signal, IEEE Transactions on Signal Processing 49 (12) (2001) 3136-3144.

[37]L. Wietzke, G. Sommer, O. Fleischmann, The signal multi-vector, Journal of Mathematical Imaging and Vision 37 (2) (2010) 132-150

[38]R. Snelick, U. Uludag, A. Mink, M. Indovina, A.K. Jain, Large-scale evaluation of multimodal biometric authentication using state-of-the-art systems, IEEE Transactions on Pattern Analysis and Machine Intelligence 27 (3) (2005) 450-455.

[39] PolyU Finger-Knuckle-Print Database, 2010. http://www.comp.polyu.edu.hk/ biometrics.

[40] J. Daugman, The importance of being random: statistical principles of iris recognition, Pattern Recognition 36 (2) (2003) 279-291.

[41] A. Martin, G. Doddington, T. Kamm, M. Ordowski, M. Przybocki, The DET cuve in assessment of detection task performance, in: Proceedings of the Eurospeech'97, 1997, pp. 1895-1898.

[42]C.D. Kuglin, D.C. Hines, The phase correlation image alignment method, in: Proceedings of the International Conference on Cybernetics and Society, 1975, pp. 163-165.

[43]B. Reddy, B. Chatterji, An FFT-based technique for translation, rotation, and scale-invariant image registration, IEEE Transactions on Image Processing 5 (8) (1996) 1266-1271. 